\title{
Acute Encephalitis Syndrome: Approach to a Changing Paradigm
}

\author{
Bidisha Banerjee ${ }^{1}$, Muhammed Hafis ${ }^{2}$, Archana Mahalingam ${ }^{3}$, Ullas Acharya ${ }^{4}$
}

\begin{abstract}
Purpose: Acute encephalitis syndrome (AES) poses challenges to physicians owing to acute presentation, often rapid neurologic deterioration, myriad causes including noninfective inflammatory disorders of central nervous system (CNS) and low microbiologic yield. We broadly discuss common and less common causes of AES and their clinical, laboratory including radiologic features as specific diagnosis guides management and improves outcome.
\end{abstract}

Materials and methods: Literature search was performed using keywords "Paediatric acute encephalitis" in MEDLINE database from 2009 to 2019 and all relevant articles (barring case reports) in English language were reviewed. Landmark articles prior to 2009 were also reviewed.

Conclusion: Acute encephalitis remains a diagnostic and therapeutic challenge in neurocritical care. The recognition of etiological agent and encephalitis mimics by investigations is important for specific therapeutic measures. Judicious use of neuroimaging, cerebrospinal fluid (CSF) analysis and appropriate lab tests helps in diagnosing specific entities especially noninfective mimics of AES which has important treatment and prognostic implication. Initial stabilization and institution of supportive measures remains key to successful management.

Keywords: Acute encephalitis, Encephalitis mimics, Etiology, Neuroimaging.

Pediatric Infectious Disease (2019): 10.5005/jp-journals-10081-1210

\section{INTRODUCTION}

Acute encephalitis syndrome (AES) is a commonly encountered problem in clinical practice. It poses challenges due to acute presentation often requiring intensive care, myriad causes from infective to noninfective inflammatory disorders of central nervous system (CNS) and low microbiologic yield. Though etiology in outbreaks of AES have been ascertained, however there is less clarity in diagnosis and management of sporadic acute encephalitis. ${ }^{1}$ Hence we broadly discuss common and less common causes of AES and their clinical, laboratory including radiologic features as specific diagnosis guides management and improves outcome.

\section{Definition}

Acute encephalitis syndrome was coined in 2008 by the World Health Organization for surveillance and research in India.

Encephalitis is characterized by an acute onset of fever and encephalopathy (altered sensorium ranging from irritability to coma) with/without seizures or other neurologic signs due to CNS inflammation. ${ }^{2}$

Encephalopathy on the other hand is a noninflammatory, often biochemical; hence, cerebrospinal fluid (CSF) shows no pleocytosis. ${ }^{2}$

\section{Causes/Etiology}

The cause varies based on the season and geographical location. Viruses have been mainly attributed to be the cause of AES in India though other microbes and toxins have been reported over the past few decades. ${ }^{1-7}$ Noninfective causes of acute encephalitic presentation are increasingly being recognized worldwide. ${ }^{8}$

Various infective and noninfective etiologies of AES have been tabulated in Table 1.

\section{Approach to Diagnosis}

Acute encephalitis syndrome is a medical emergency. Though the causes of acute encephalitis are many, initial presentation may be similar. High index of suspicion with awareness of "encephalitis mimics," prompt, focused clinical evaluation and initial stabilization
${ }^{1-4}$ Manipal Hospitals, Bengaluru, Karnataka, India

Corresponding Author: Bidisha Banerjee, Manipal Hospitals, Bengaluru, Karnataka, India, Phone: +91 9980946290, e-mail: drbidisha@yahoo.co.in

How to cite this article: Banerjee B, Hafis M, Mahalingam A, et al. Acute Encephalitis Syndrome: Approach to a Changing Paradigm. Pediatr Inf Dis 2019;1(3):86-94.

Source of support: Nil

Conflict of interest: None

are corner stone of management. Identification of a specific etiology is guided by the observations of astute clinicians and supported by laboratory data and neuroradiology.

\section{Key Points in History ${ }^{10}$}

- Fever

- Duration and progression of symptoms (seizure, psychiatric, other systemic)

- Change in behavior/cognition/personality or altered consciousness

- New onset seizure

- Focal neurological symptoms.

- Etiologic clues Demography/outbreaks/travel

Recent vaccination (acute disseminated encephalomyelitis (ADEM))

Contact with animals (rabies), consumption of raw milk (brucellosis)

Contact with unchlorinated fresh water (amoeba) contaminated with animal urine (leptospirosis) and exposure to mosquito/tick bites (arboviruses, Lyme disease, tick borne encephalitis)

Known risk factors for HIV/immunocompromised.

Pre-illness epilepsy/developmental status

Siblings with similar illness/multiple episodes of encephalopathy: classically seen in some inborn errors of metabolism. 
Table 1: Varied etiologies of acute encephalitis syndrome $e^{9,10}$

Infective $\left\{\begin{array}{l}\hline \text { Viral-HSV, JE, entero, } \\ \text { parechovirus in neonates } \\ \text { Bacterial-ABM } \\ \text { - Tubercular meningitis } \\ \cdot \text { Dengue } \\ - \text { Neurobrucellosis } \\ - \text { Enteric } \\ \cdot \text { Rickettsial } \\ \cdot \text { Malaria }\end{array}\right.$

Noninfective $\left\{\begin{array}{l}\text { - Immune-mediated } \\ \text { - ADEM/NMOSD } \\ \text { - Autoimmune encephalitis-anti NMDAR, } \\ \text { limbic encephalitis } \\ \text { - Hashimoto's encephalopathy } \\ \text { - Bickerstaff brainstem encephalitis } \\ \text { - Probable inflammatory } \\ \text { - Acute necrotizing encephalopathy (ANE) } \\ \text { - FIRES (Febrile infection-related epilepsy } \\ \text { syndrome) } \\ \text { - Others } \\ \text { - IEM (Inborn errors of metabolism) } \\ \text { - NCSE (Nonconvulsive status epilepticus) }\end{array}\right.$

\section{Key Points in Examination ${ }^{10}$}

- Airway, breathing, circulation, neurologic disability (ABCD) [Glasgow coma scale (GCS), pupils, signs of impending brain herniation]

- General physical examination-pallor, icterus, skin rashes, oarotid swelling/orchitis, labial herpes, bite marks*

- Systemic examination a. Hepatosplenomegaly, lymphadenopathy-TB, Brucella

- Neurological examination-level of consciousness, signs of impending brain herniation, signs of meningeal irritation, focal neurologic deficit, evidence of subtle motor seizures (twithing of mouth, eyelid, digit), papilledema, flaccid paralysis (anterior horn cell involvement), movement disorders, including parkinsonism

Peripheral blood can show relative lymphocytosis in viral encephalitis. Peripheral blood smear (thick and thin smear) is the most sensitive and specific test for cerebral malaria. Cytoplasmic inclusions in the peripheral blood monocytes are characteristic of ehrlichiosis, $10 \%$ of these children develop meningoencephalitis. Rickettsial infection and viral hemorrhagic fevers characteristically show the presence of leukopenia and thrombocytopenia. Mycoplasma, legionella or tuberculosis infections can be diagnosed by the characteristic findings in chest radiography. Blood glucose, kidney and liver function tests will help to pick-up metabolic encephalopathy (Table 2).

Identification of etiological agent is necessary for prompt initiation of specific therapy, epidemiology, identifying targets

*Pallor in cerebral malaria/intracranial bleed. Icterus in leptospirosis, cerebral malaria, hepatic encephalopathy. Skin rashes-meningococcemia (purpuric), dengue (face/blanching), measles (erythematous maculopapular starting from face), varicella (vesicular/trunk), rickettsial (papules on palms and soles), arboviral and enteroviral (vesicular in HFMD). Parotid swelling/orchitis in mumps. Labial herpes in herpes simplex virus encephalitis.
Table 2: Lab diagnostic evaluation in acute encephalitis syndrome

\begin{tabular}{ll}
\hline In all & $\begin{array}{l}\text { Complete blood count, blood culture, electrolytes/ } \\
\text { blood glucose, liver and kidney function tests, } \\
\text { peripheral smear for malarial parasite, chest X-ray }\end{array}$ \\
In most & $\begin{array}{l}\text { Neuroimaging, CSF analysis after stabilization if no } \\
\text { contraindications*, EEG if seizure or suspicion of } \\
\text { non-convulsive status epilepticus (NCSE) or } \\
\text { autoimmune encephalitis (AE) }\end{array}$ \\
In some & $\begin{array}{l}\text { Autoimmune work up-antineuronal (NMDAR, VGKC), } \\
\text { anti-thyroid microsomal/thyroglobulin antibodies, ANA, } \\
\text { ESR, neuromyelitis optica (NMO) and myelin } \\
\text { oligodendrocyte glycoprotein (MOG IgG) in serum } \\
\text { IEM screen-ABG, plasma lactate, ammonia, urine } \\
\text { ketones, dried blood spot for tandem mass } \\
\text { spectrometry (TMS), urine gas chromatography mass } \\
\text { spectrometry (GCMS) }\end{array}$ \\
\hline
\end{tabular}

*Contraindications to lumbar puncture include hemodynamic instability, features of raised intracranial pressure, bleeding diathesis, local site infection). A neuroimaging study is done if lumbar puncture is contraindicated

for immunization, charting preventive strategies, implementing appropriate control measures especially in outbreak situations, and to formulate rational empirical treatment especially for nonviral causes of AES. However, there are challenges in the rapid identification of the etiological agent. Firstly, the number of infectious pathogens known to cause AES is sizeable; including several viruses, bacteria, fungi, and protozoa. Secondly, several conventional methods available for testing are time-consuming, expensive, lack adequate sensitivity, and/or specificity and may not be easily accessible. Thirdly, the small volume of CSF available from patients is quite often insufficient for laboratory testing for all the pathogens. ${ }^{11}$

Cerebrospinal fluid should be collected in sterile, screw capped containers and immediately stored at $2-8^{\circ} \mathrm{C}$ until testing. If a delay in testing is expected, CSF samples must be frozen at $-20^{\circ}$. If testing for bacterial pathogens, the CSF must not be refrigerated. ${ }^{11}$ Additional samples may be stored for antibody testing if needed. The CSF is examined for cytology, biochemistry, Gram's stain, wet mount for motile amoeba, ZiehlNeelsen stain for acid fast bacilli, PCR for HSV 1 and 2, nucleic acid amplification test (NAAT) for MTB, bacterial culture, IgM antibodies for JE and dengue virus if suspected. Multiplex PCR may be performed wherever feasible esp. if already received antimicrobials. Blood sugar has to be checked just prior to the lumbar puncture to measure the CSF to blood sugar ratio.

Cerebrospinal fluid analysis may provide invaluable information about the nature of the infectious process. Lymphocytic pleocytosis ( $>5$ lymphocytes $/ \mathrm{mm}^{3}$ ) is present in $>95 \%$ of the cases along with normal glucose and protein or mildly raised protein in viral encephalitis indicating meningeal inflammation. Alternative etiology (encephalopathy) is considered if CSF lymphocytosis is absent. Delay in analysis of the CSF sample is avoided because the cells in CSF lyse during storage and transport of the sample. An inflammatory response may not be seen in the initial stages of atypical HSE and among immunocompromised (by cancer chemotherapy or irradiation).

Other specimens which may be collected depending on the clinical presentation and suspected etiology of AES are nasopharyngeal/throat swabs, swabs from vesicles, rectal swabs/ stool specimens, urine and brain biopsy. 11,12

Table 3 CSF analysis in various infective causes of AES (Table 4). 
Table 3: Treatment options in autoimmune encephalitis

\begin{tabular}{llll}
\hline & Treatment options & Dose and schedule & Duration \\
\hline First line & IV methyl prednisolone & $20-30 \mathrm{mg} / \mathrm{kg} /$ day & $3-5$ days \\
& $\begin{array}{l}\text { Intravenous immunoglobulin } 2-5 \text { days } \\
\text { Plasmapheresis }\end{array}$ & $2 \mathrm{~g} / \mathrm{kg}$ in divided doses & $5-7 \mathrm{cycles}$ \\
Cyclophosphamide & & $750 \mathrm{mg} / \mathrm{m}^{2}$ \\
Recond line & Rituximab & $375 \mathrm{mg} / \mathrm{m}^{2}$ followed by second dose after \\
& 2 weeks & \\
\hline
\end{tabular}

Table 4: CSF analysis in various infective causes of AES

\begin{tabular}{|c|c|c|c|c|c|}
\hline Investigation & Normal & Bacterial & Viral & Tuberculosis & Fungal \\
\hline Opening Pressure & $10-20 \mathrm{~cm}$ & High & Normal/high & High & High/very high \\
\hline Color & Clear & Cloudy & “Gin” color & Cloudy/yellow & Clear/cloudy \\
\hline \multirow[t]{2}{*}{ Cells } & $<5$ & High/very high & Slightly increased & Slightly increased & Normal-high \\
\hline & & $100-50,000$ & $5-1,000$ & $<500$ & $0-1,000$ \\
\hline Differential & Lymphocytes & Neutrophils & Lymphocytes & Lymphocytes & Lymphocytes \\
\hline CSF/plasma glucose & $50-66 \%$ & Low $<40 \%$ & Normal & Low-very low (<30\%) & Normal-low \\
\hline \multirow[t]{2}{*}{ Protein (g/L) } & $<0.45$ & High & Normal-high & High-very high & Normal-high \\
\hline & & $>1$ & $0.5-1$ & $1.0-5.0$ & $0.2-5.0$ \\
\hline
\end{tabular}

\section{Acute encephalitis syndrome-neuroimaging}

Neuroimaging should be done in all; magnetic resonance imaging (MRI) is preferred over CT as it is more sensitive however where imaging needs to be urgently performed, intracranial bleed suspected and sedation not possible, contrast enhanced CT scan may be obtained. Use of limited sequences on MRI like diffusion and fluid-attenuated inversion recovery (FLAIR) and compressed sense imaging softwares can reduce scan times remarkably (to $<5$ minutes).

\section{Role of Neuroimaging}

- Excludes encephalitis mimics ${ }^{\dagger}$

- Presence and extent of inflammation, radiologic signs of raised intracranial pressure ${ }^{\ddagger}$

- Etiologic clues in some based on "pattern recognition" (Figs 1 to 3 )

${ }^{\dagger}$ Helps in diagnosis of specific entities like TBM, ADEM, ANE, AESD, MERS with therapeutic and prognostic significance.

₹Presence of midline shift, obliteration of basal cisterns and slit ventricles are radiological contra-indications of $L P$.

In MRI, FLAIR sequences are useful for detection of vasogenic edema, diffusion weighted images for cytotoxic edema and post gadolinium for meningeal enhancement/inflammation, ${ }^{9}$ susceptibility weighted imaging for mico/macro bleeds and vasculitic infarcts.

"Typical geographic patterns and etiology.,10

\section{Affected Region-Etiology}

Cortical gray (medial temporal, orbito-frontal, insular, cingulate)— HSV

- Basal ganglia/thalamus-Japanese encephalitis, West Nile

- Brain stem and spinal cord (anterior horn cells) —enterovirus

- Cerebellum-mycoplasma, varicella zoster virus, dengue, scrub typhus, rotavirus

\section{Encephalitis Mimics}

Diagnosis of Tubercular Meningitis ${ }^{13}$

- Clinical-fever (>5 days), headache, vomiting, altered sensorium/focal neurologic deficit
- Examination of CSF reveals lymphocytic pleocytosis (10-500 $\mu \mathrm{L},>50 \%)$ with raised proteins $>100 \mathrm{mg} / \mathrm{dL}$, low sugar $(20-40$ $\mathrm{mg} / \mathrm{dL}$ ) and/or positive ZN stain, acid fast bacilli (AFB) culture, PCR for MTB

- Imaging reveals basal meningeal enhancement, tuberculoma, hydrocephalus, infarct, pre-contrast basal hyperdensity

- Evidence of TB in other sites (Fig. 4).

Outcome depends on early identification (when symptoms are non-specific) and adequate treatment. ${ }^{14}$

MRC staging of TBM consists of 3 stages with increasing severity

- prodrome, no neurologic signs and symptoms

- meningeal signs, normal sensorium, cranial nerve palsy \pm

- altered sensorium, seizures, focal neurodeficits/involuntary movements.

\section{Acute Necrotizing Encephalopathy of Childhood}

Suspect if fever (esp. viral illness) followed by rapid neurologic deterioration (sensorium/tone/raised ICP). Management includes, stabilization, neuroimage (CT/MRI brain which typically show multiple bilateral brain lesions in thalami or putamina, internal capsule of the cerebrum or white matter of the cerebellum and tegmentum (Fig. 5). ${ }^{15}$ Definite treatment guidelines are not available, however standard supportive care, immunotherapy-usually high dose pulse methylprednisolone, therapeutic hypothermia before 12 hours (decreases cytokines) and anti-edema measures are beneficial. ${ }^{16}$ Outcomes in acute necrotizing encephalopathy (ANE) are heterogeneous but potentially devastating with $30 \%$ mortality and $10 \%$ intact survival. Predictors of outcome include, age ( $>4$ years, $<1$ year), increased CSF protein/transaminases, presence of hemorrhage/cavitation/brainstem lesions on neuroimaging and ANE-severity score. ${ }^{17}$

\section{Acute Disseminated Encephalomyelitis}

Acute disseminated encephalomyelitis is first polyfocal clinical event affecting CNS characterized by encephalopathy not due to fever/post ictal phase/systemic illness. Brain MRI shows diffuse, 

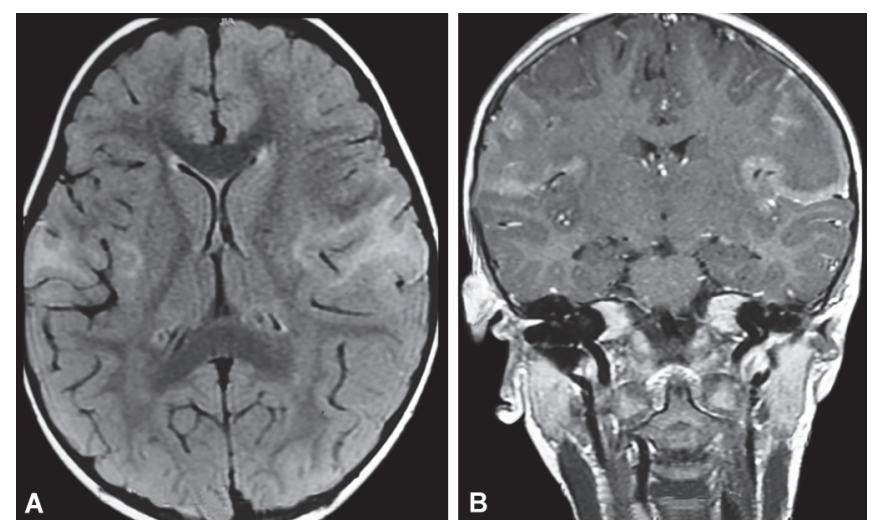

Figs 1 A and B: Herpes simplex encephalitis (HSE): (A) Axial FLAIR showing bilateral periSylvian cortex hyperintense signals; (B) Coronal T1 post-gad shows focal enhancement of the same

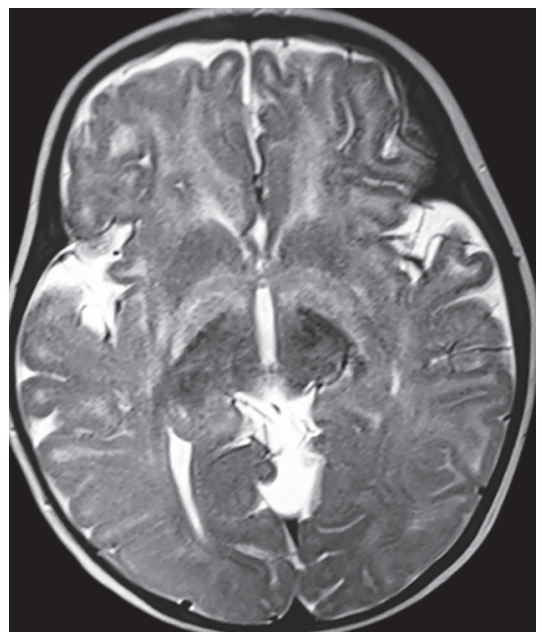

Fig. 3: Glutaric aciduria type I-Axial T2 weighted image shows frontotemporal atrophy with widened Sylvian fissure (Bat's wing appearance) and hyperintense signals in bilateral globus pallidi, putamina
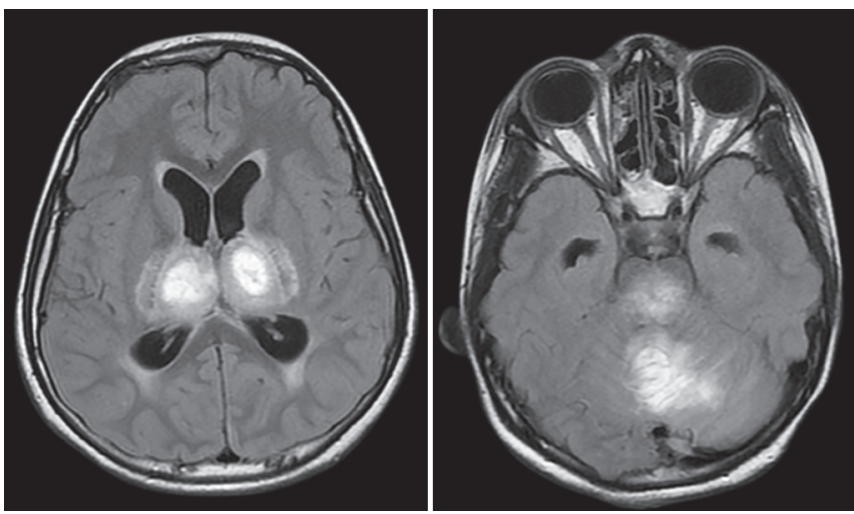

Fig. 5: ANE-Axial FLAIR images showing symmetric hyperintensities in bilateral thalami, brainstem and cerebellar white matter

poorly demarcated, large white mater lesions with or without lesions in deep grey/spinal cord. There should be no new clinical symptoms and MRI abnormalities (Fig. 6) after the first 3 months from symptom onset. ${ }^{18}$

Long tract (pyramidal) signs, cranial nerve palsies, meningism, signs of opticospinal involvement may be seen in addition to encephalopathy. ${ }^{19}$
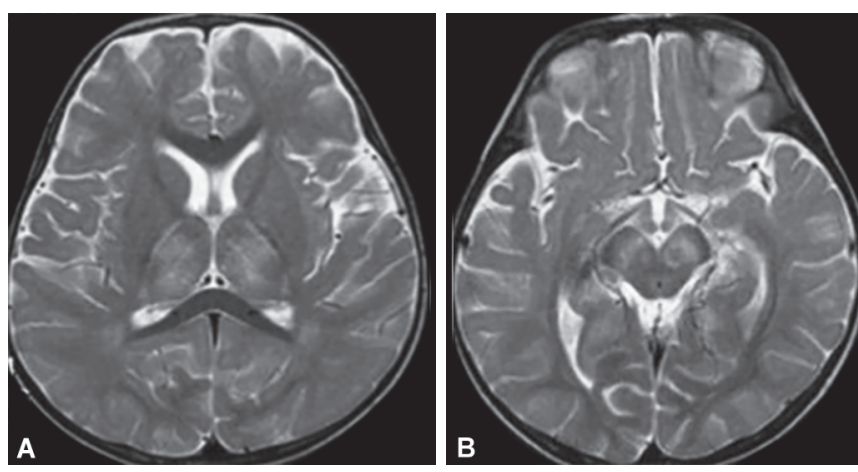

Figs $2 A$ and B: Japanese encephalitis-Axial T2 weighted images showing (A) Bilateral thalamic; (B) Bilateral substantia nigra hyperintensities
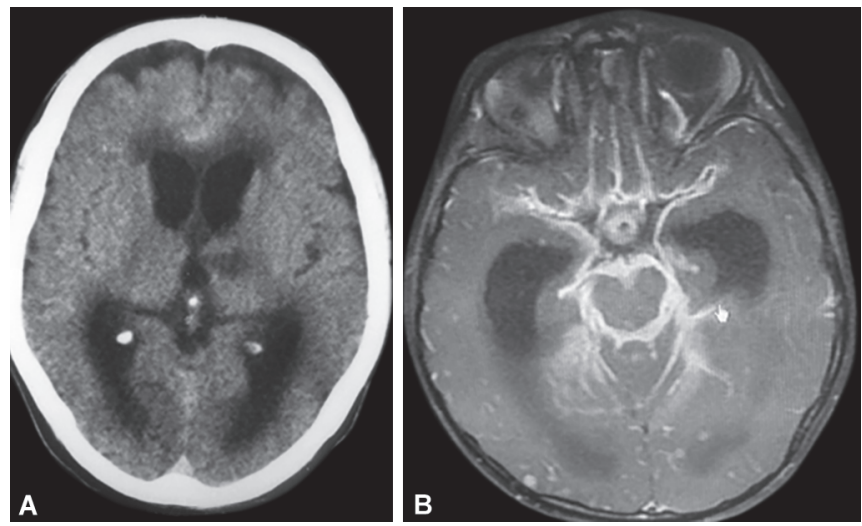

Figs 4A and B: TBM: (A) Plain CT head showing hydrocephalus with left thalamic hypodensity (infarct); (B) Gad-enhanced MRI brain showing basal exudates

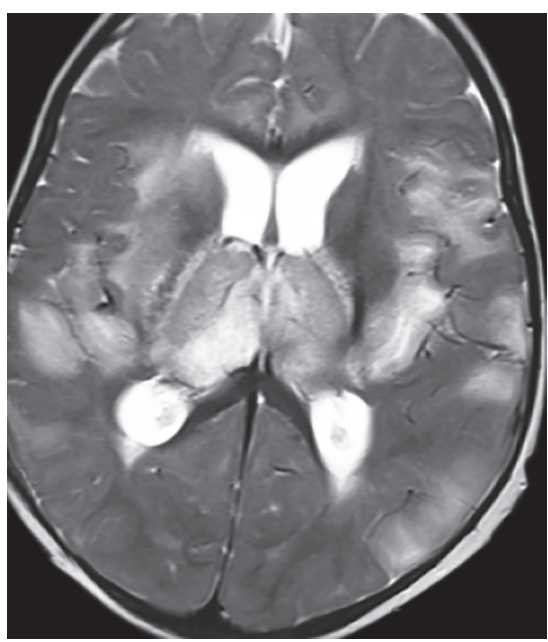

Fig. 6: ADEM-AxialT2 weighted image showing asymmetric, multifocal, subcortical white matter and bilateral thalamic hyperintensities

Cerebrospinal fluid may show pleocytosis with raised proteins; oligoclonal bands might be present in a small subset. Where appropriate serum antibodies to myelin oligodendrocyte glycoprotein (MOG IgG) and neuromyelitis optica (NMO) IgG/antiaquaporin antibodies may be obtained.

Treatment is with high dose pulse methylprednisolone of 30 $\mathrm{mg} / \mathrm{kg} /$ day, with a maximum dose of $1 \mathrm{~g} /$ day over $3-5$ days followed by oral steroid taper. Prognosis is generally favorable. ${ }^{18}$ Autoimmune encephalitis (AE) - clinical clues include, ${ }^{20}$ 
- Acute/subacute neurologic dysfunction (altered sensorium, cognition, speech)

- Seizures-facio brachial dystonic in LGI1

- Behavioural/psychiatric disturbance

- Movement disorderorofacial dyskinesia, catatonia

- Insomnia/somnolence

- Autonomic-central hypoventilation

Probable anti-N-methyl-D-aspartate (NMDA) receptor encephalitis-diagnosis is made when all of the three criteria are met:

- Rapid onset (<3 months) of at least four of the six following major groups of symptoms:

- Abnormal (psychiatric) behavior or cognitive dysfunction Speech dysfunction mutism, verbal reduction, pressured speech)

Seizures

Movement disorder, dyskinesia or rigidity/abnormal posture

Decreased consciousness/autonomic dysfunction or central hypoventilation

At least one of the following laboratory study results

Abnormal electroencephalogram (EEG) (focal/diffuse slow/ disorganised activity/epileptic activity/extreme delta brush)

Cerebrospinal fluid with pleocytosis or oligoclonal bands

Reasonable exclusion of other disorders

Diagnosis can be made with any of the 3 of the above group of symptoms along with a systemic teratoma

Definite anti-NMDA receptor encephalitis:

Diagnosis is made in the presence of one or more of the 6 major groups of symptoms and IgG anti-GluN1 antibodies, after reasonable exclusion of other disorders.

\section{Anti-NMDAR Encephalitis (Anti-NMDARE)}

It is "A multistage illness that progresses from psychosis, memory deficits, seizures, and language disintegration into a state of unresponsiveness with catatonic features often with abnormal movements and autonomic and breathing instability." Children/ young adults are affected predominantly. It can occur with or without tumor (usually ovarian teratoma in women $>18$ years), patients with tumor resection and immunotherapy respond faster, less often need 2 line immunotherapy in comparison with patient without a tumor. Substantial recovery is seen in around $75 \%$ of all patients in inverse order of symptom development, associated with a decline of antibody titers. First line treatment is pulse methylprednisolone and IVIG. Second line being rituximab/ cyclophosphamide (lack of response to 1st line within 1 month). ${ }^{21}$ Usually LGI1 respond to steroids alone. Anti-N-methyl-D-aspartate receptor (NMDAR) needs more intensive immunotherapy. End point is clinical response. There is no consensus on duration of treatment especially for long-term immunosuppression which is individualized according to benefits/risks.

\section{Diagnostic Criteria for Definite Autoimmune Limbic Encephalitis}

Diagnosis can be made when all four of the following criteria have been met:

- Subacute onset (rapid progression of less than 3 months) of working memory deficits, seizures, or psychiatric symptoms suggesting involvement of the limbic system
- Bilateral brain abnormalities on $T_{2}$-weighted/fluid-attenuated inversion recovery MRI highly restricted to the medial temporal lobest

At least one of the following

Cerebrospinal fluid pleocytosis (WBC count $>5$ cells $/ \mathrm{mm}^{3}$ ) EEG with epileptic or slow-wave activity involving the temporal lobes

Reasonable exclusion of alternative causes

\section{Diagnostic Criteria for Probable (Seronegative) Autoimmune Encephalitis}

Diagnosis can be made when all four of the following criteria have been met:

- Rapid progression in less than 3 months with short-term memory loss, altered mental status or psychiatric symptoms

- Well defined syndromes of autoimmune encephalitis like typical limbic encephalitis, Bickerstaff's brainstem encephalitis and ADEM have to be excluded

- Absence of well characterized autoantibodies in the serum and CSF and at least 2 of the following criteria:

- MRI abnormalities suggestive of autoimmune encephalitis

- Cerebrospinal fluid pleocytosis

- Cerebrospinal fluid specific oligoclonal bands or elevated CSF IgG index or both

- Inflammatory infiltrates in the brain biopsy and excluding other disorders like tumor

- Reasonable exclusion of alternate causes

\section{Less Common Causes of AES}

Hashimoto encephalopathy $(\mathrm{HE})$ is characterized by encephalopathy with presence of thyroid microsomal antibody and good response to glucocorticoid. The clinical presentations in HE is variable, so diagnosis has to be highly considered when such a presentation is present. Presentation in some is dramatic with acute onset unexplained seizures resistant to anti-epileptic dugs, confusion, hallucinations, impairment of cognitive function, behavioral and mood disturbances, disturbance of consciousness, headaches, stroke-like episodes, focal neurodeficits, ataxia and presence of high thyroid antibody levels, esp. TPO Ab. Pathogenesis is not clear, however vasculitis and autoimmunity are thought to be causal. Thyroid function is normal/decreased; rarely increased. To rule out other etiologies CSF, EEG, MRI brain are needed. Once diagnosis made, corticosteroids provide dramatic recovery. ${ }^{22}$

\section{Bickersta's Brainstem Encephalitis}

When both the criteria given below are fulfilled the diagnosis of Bickerstaff brainstem encephalitis is made: (1) subacute onset (rapid progression of less than 4 weeks) of all the following symptoms: decreased level of consciousness, bilateral external ophthalmoplegia, ataxia (2) alternative causes has to be reasonably excluded. ${ }^{20,23}$

Definite diagnosis of Bickersta's brainstem encephalitis can be made with positive IgG anti-GQ1b antibodies even if there is no complete bilateral external ophthalmoplegia or ataxia cannot be assessed. ${ }^{20}$

FIRES is "Febrile Infection Related Epilepsy Syndrome." It is defined as "a subtype of new onset refractory status epilepticus (NORSE) that requires a prior febrile infection, with fever starting between 2 weeks 
and 24 hours prior to onset of refractory SE, with or without fever at onset of SE." This definition excludes prolonged febrile seizures. ${ }^{24} \mathrm{FIRES}$ includes all ages. It is an immune disorder triggered by an infection and it affects the brain with an intrinsic predisposition toward an autosustaining epileptogenic process. These are suggested by its biphasic clinical course. In this otherwise drug refractory condition ketogenic diet (KD), therapeutic hypothermia, cannabidiol (CBD), anakinra (recombinant, modified human interleukin-1 receptor antagonist) and continuous intravenous administration of magnesium sulfate have provided therapeutic benefit. ${ }^{25}$

New-onset refractory status epilepticus is not a specific diagnosis. It is a clinical presentation in a patient with new onset refractory status epilepticus, with no other relevant neurological disorder/active epilepsy, without any acute/active structural o toxic or metabolic cause.

\section{Acute Encephalopathy with Biphasic Seizures and Late Reduced Diffusion (AESD)}

The initial neurological symptom on day 1 is a febrile seizure (usually $>30$ minutes). This is followed by secondary seizures at day $4-6$. It is reported only in East Asian infants. A variable level of neurological sequelae is displayed among these children. MRI can be normal with no abnormality in first two days; frontal or fronto-parietal subcortical white matter shows reduced diffusion during days 3-9, then disappears between days 9 and days 25 . Excitotoxic injury with delayed neuronal death is hypothesized as a possible mechanism based on MR spectroscopic findings. ${ }^{26}$ The clinical course of acute encephalopathy can be improved by early administration of vitamins (B1, B6, and L-carnitine). Favorable results can be obtained by mitochondrial rescue and neuroprotection. ${ }^{27}$

\section{Clinically Mild Encephalitis/Encephalopathy with a Reversible Splenial Lesion (MERS)}

It is a reversible lesion with homogeneously reduced diffusion in the corpus callosum (at least involving the splenium). It can be associated with symmetrical white matter lesions. Delirious behavior is the most common neurological symptom. It is followed by disturbance in consciousness, seizures. These symptoms completely recover within a month. Intramyelinic edema, interstitial oedema in tightly packed fibers, and a transient inflammatory infiltrate are the postulated reasons for the transiently reduced diffusion within the lesions. ${ }^{26}$

Clinical pointers of an underlying IEM in AES include unexplained rapidly progressive encephalopathy and seizures, recurrent episodes of encephalopathy/ataxia especially with intercurrent illness, persistent vomiting or failure to thrive, consanguinity/family history of neonatal/infant deaths, alopecia and seborrheic dermatitis, periorificial rash and high anion gap metabolic acidosis, hypoglycaemia. ${ }^{28}$

\section{Management}

It should focus on initial stabilization, arriving at a specific diagnosis and preventing secondary brain injury. Though variety of infective and noninfective etiologies are implicated in AES, initial stabilization should precede, a targeted approach (antimicrobials and/or immunotherapy) for specific etiology (Table 5).

The mortality and morbidity associated with AES can be reduced by stabilizing the airway, breathing and circulation along with other supportive care measures. Patients are managed in intensive care unit when the GCS $<8$ with features of raised intracranial pressure, status epilepticus and shock. Supportive care includes, maintaining euvolemia and normoglycemia, and preventing hyponatremia by isotonic maintenance intravenous fluids. Hypoglycemia and hyperglycemia should be avoided by regular monitoring of blood glucose. Hyperthermia is to be avoided., ${ }^{9,12}$

Prompt recognition and management of raised ICP is important. Reduced mean arterial pressure (MAP) or raised intracranial pressure (ICP) or both can reduce the cerebral perfusion pressure (CPP) (CPP = MAP-ICP) and hence the CPP has to be maintained. Intubation and ventilation is considered (if GCS $<8$, or evidence of irregular

Table 5: Summary of antiviral, antibiotics and antifungal agents

\begin{tabular}{|c|c|c|c|}
\hline Indication & Choice of drug & Dose and schedule & Duration \\
\hline HSV & Acyclovir & 3 months to 12 years: $500 \mathrm{mg} / \mathrm{m}^{2}$ every 8 th hourly & 14-21 days \\
\hline VZV & & Above 12 years: $10 \mathrm{mg} / \mathrm{kg}$ every 8 th hourly & \\
\hline Meningococci & Ceftriaxone/cefotaxime + vancomycin & 100 mg/kg/day & 10-14 days \\
\hline \multirow[t]{2}{*}{ Pneumococci } & & $200 \mathrm{mg} / \mathrm{kg} /$ day & \\
\hline & & $60 \mathrm{mg} / \mathrm{kg} /$ day & \\
\hline \multirow[t]{3}{*}{ Salmonella } & Ceftriaxone/cefotaxime/meropenem & $100 \mathrm{mg} / \mathrm{kg} /$ day & $\begin{array}{l}4 \text { weeks (up to } 6 \text { weeks } \\
\text { in presence of abscess) }\end{array}$ \\
\hline & & 200 mg/kg/day & \\
\hline & & $40 \mathrm{mg} / \mathrm{kg} / \mathrm{dose}$ 8th hourly & \\
\hline \multirow[t]{3}{*}{ Brucella } & Doxycycline + rifampicin + ceftriaxone & $2-4 \mathrm{mg} / \mathrm{kg} / \mathrm{day}$ & 4-6 months \\
\hline & & $15-20 \mathrm{mg} / \mathrm{kg} / \mathrm{day}$ & $4-6$ months \\
\hline & & $100 \mathrm{mg} / \mathrm{kg} /$ day & 4 weeks \\
\hline \multirow[t]{2}{*}{ Rickettsia } & Doxycycline & 4 mg/kg/day & $5-7$ days \\
\hline & Azithromycin* & $10 \mathrm{mg} / \mathrm{kg} / \mathrm{day}$ & 5 days \\
\hline \multirow[t]{5}{*}{ Cryptococcus } & Induction: amphotericin B + flucytosine & $0.7-1 \mathrm{mg} / \mathrm{kg} / \mathrm{day}$ & 2 weeks \\
\hline & $\begin{array}{l}\text { Maintenance therapy: fluconazole/ } \\
\text { amphotericin B flucytosine }\end{array}$ & 100 mg/kg/day & 2 weeks \\
\hline & & 12 mg/kg/day & 10 weeks \\
\hline & & $0.7-1 \mathrm{mg} / \mathrm{kg} /$ day & $6-10$ weeks \\
\hline & & 100 mg/kg/day & \\
\hline
\end{tabular}


respirations and inability to maintain airway or herniation) to maintain normocapnia and avoid hypoxemia. Adequate analgesia and sedation has to be provided. Avoid noxious stimuli; prior to endotracheal tube suctioning, ET lignocaine should be administered. ${ }^{12}$

Osmotherapy in form of hypertonic saline and/or mannitol may be used when clinical evidence of raised ICP is present. The patient is hyperventilated to maintain a target of $\mathrm{PaCO}_{2}-30-35 \mathrm{~mm} \mathrm{Hg}$, when there are signs of impending herniation. Mannitol is given at a dose of initial bolus of $0.25 \mathrm{~g} / \mathrm{kg}$, then $0.25 \mathrm{~g} / \mathrm{kg}, q 6$ hours up to 48 hours. In the presence of hypotension, hypovolemia, and renal failure, hypertonic (3\%) saline is preferred to mannitol. It is given at a dose of $0.1-1 \mathrm{~mL} / \mathrm{kg} / \mathrm{hour}$ by infusion and the serum sodium should be targeted to a level of $145-155 \mathrm{mEq} / \mathrm{L}$. ${ }^{9,12}$

The seizures have to be controlled with anticonvulsant in the background of GCS $<8$, and features of raised ICP, as seizures may further raise the intracranial pressure.

A benzodiazepine is used to abort clinical seizures (Lorazepam $0.1 \mathrm{mg} / \mathrm{kg}$ or midazolam $0.1 \mathrm{mg} / \mathrm{kg}$ ) followed by loading dose of fosphenytoin (30 mg/kg).

\section{Antimicrobials}

Empirical treatment with broad spectrum antibiotic must be started while awaiting lab investigations. Acute meningoencephalitis due to common bacterial pathogens may be treated with 3 rd generation cephalosporins, ceftriaxone or cefotaxime. ${ }^{12}$ In suspected pneumococcal meningitis vancomycin ( $60 \mathrm{mg} / \mathrm{kg} /$ day in 3-4 divided dose) should be added to 3rd generation cephalosporin. Crystalline penicillin is the drug of choice for Neisseria infection; however, ceftriaxone or cefotaxime are acceptable alternatives in case of non-availability or resistance. All cases of sporadic viral encephalitis must be started on acyclovir, as outcomes in HSE depend on early and adequate treatment., ${ }^{9,10}$ When an alternative diagnosis has been made, or HSV PCR in the CSF is negative and MRI is normal acyclovir should be stopped. However, if the CSF PCR for HSV or MRI may be falsely negative, when performed within 48 hours of symptom onset. These studies should be repeated before stopping acyclovir if the clinical suspicion of HSE is high. When there is a suspicion of cerebral malaria, empirical anti-malarial (artemisinin-based combination therapy) must be started. This should be stopped if the peripheral smear and rapid diagnostic tests are negative. Neurobrucellosis is treated with triple drug therapy including doxycycline, ceftriaxone and rifampicin. ${ }^{29} \mathrm{~A}$ high index of suspicion of rickettsial encephalitis should be there if from an endemic area with febrile illness with or without rashes. Doxycycline is considered to be the drug of choice; azithromycin can be used as an alternative in doxycycline resistant or contraindicated patients. ${ }^{30}$ In primary and granulomatous amoebic meningoencephalitis rifampicin, co-trimoxazole, azithromycin and fluconazole has been used with reported efficacy of miltefosine. ${ }^{31,32}$ Evidence for treatment of mycoplasma in AES is poor, however when needed azithromycin, doxycycline or fluoroquinolones may be used. ${ }^{9}$ Pleconaril has been found to be useful in enterovirus encephalitis except in EV71. ${ }^{33}$ There is experimental evidence of benefit of minocycline in $\mathrm{JE}^{34}$

\section{Antitubercular agents}

Antitubercular treatment (ATT) for 10-12 months is recommended for CNS tuberculosis by RNTCP. In a new case with CNS TB or drug sensitive case previously treated with ATT, initial 2 month of therapy is with isoniazid, rifampicin, pyrazinamide and ethambutol followed by $8-10$ months of isoniazid, rifampicin and ethambutol as daily regimens. ${ }^{35}$ Strict compliance and adherence to treatment and monitoring is paramount in any tuberculosis case.

\section{Immunotherapy}

Indications: Steroids are used in ADEM, Hashimoto encephalopathy, and autoimmune encephalitis. ${ }^{19,21}$ In Bickerstaff encephalitis, $A D E M$ and $A E$, IVIG is also used. Usage of corticosteroids in viral encephalitis is not established but may be considered along with acyclovir in patients with marked cerebral edema or raised ICP. Though the clinical benefit is not well established, IVIG has been used in EV 71 encephalitis (Table 3). ${ }^{33}$

\section{Corticosteroids}

Corticosteroids have been used in therapy of both infective and noninfective encephalitis. It is a widely accepted first line pharmacotherapy of ADEM and other immune mediated encephalitis syndromes. Pulse doses of methyl prednisolone (20-30 mg/kg/day) with a maximum dose of $1 \mathrm{~g} /$ day for 3-5 days is recommended for ADEM and other immune mediated encephalitis syndromes (anti-NMDAR encephalitis, anti-VGKC encephalitis, Hashimoto's encephalopathy, Bickerstaff encephalitis, etc.). ${ }^{18,19}$ Pulse steroids should be followed with low dose (1-2 mg/kg/day) oral prednisolone to taper over 4-6 weeks in ADEM and possibly longer in AE. Systemic steroids are recommended in the treatment of CNS TB. Prednisolone $2 \mathrm{mg} / \mathrm{kg} /$ day or dexamethasone $0.6 \mathrm{mg} / \mathrm{kg} /$ day in divided doses for at least 4 weeks and then tapering over next 4 weeks is recommended. It is also recommended in enteric encephalopathy.

\section{Intravenous Immunoglobulins}

Intravenous immunoglobulin (IVIG) is an alternative/adjunctive option for the treatment of ADEM and other immune mediated encephalitis. IVIG in a dose of $2 \mathrm{~g} / \mathrm{kg}$ divided over $2-5$ days is used. Intravenous immunoglobulin is preferred mode of treatment in Bickerstaff brainstem encephalitis (GBS variant).

\section{Plasmapheresis}

Plasmapheresis is another first line treatment option for immune mediated encephalitis; 5-7 exchanges every other day showed favorable outcome. Technical expertise and monitoring for hemodynamic instability make plasmapheresis difficult compared to IVIG especially in a younger child.

\section{Other Immunosuppressant Drugs}

Treatment with other immunosuppressant medicine like rituximab or cyclophosphamide should be considered in immune mediated encephalitis, which is refractory to steroid, IVIG and plasmapheresis. ${ }^{21}$ Cyclophosphamide in a dose of $750 \mathrm{mg} / \mathrm{m}^{2}$ body surface area intravenously can be given. Good hydration and Mesna should be provided to eliminate the risk of cyclophosphamide induced hemorrhagic cystitis. Cyclophosphamide is known to cause bone marrow suppression, hence a complete hemogram is advisable. Rituximab, is a monoclonal antibody which acts by inhibiting CD-20 also preferred as 2 nd line treatment in autoimmune encephalitis. Rituximab in a dose of $375 \mathrm{mg} / \mathrm{m}^{2}$ followed by a second dose after 2 weeks is recommended.

\section{Acute Management of IEM}

Aims of treatment are to

- Decrease the substrate (stop feeds) to reduce the formation of toxic metabolites

- Provide adequate calories and prevent endogenous catabolism

- Enhance the excretion of toxic metabolites

- Give co-factor therapy for specific disease or empirically if the diagnosis is not established 


\section{Supportive Care}

Treat seizures (but avoid topiramate, valproate, zonisamide), fluid, electrolyte and acid base balance, maintain normothermia and euglycemia, treat infection and mechanical ventilation.

Hyperammonemia is managed by

- Discontinuation of feeds. IV glucose and lipids are given to provide adequate calories (GIR $-8-10 \mathrm{mg} / \mathrm{kg} / \mathrm{minute}$ and intralipid $-0.5-3 \mathrm{~g} / \mathrm{kg} /$ day). Protein is added gradually after stabilization (0.25-1.5 g/kg/day).

- Rapid removal of ammonia by dialysis (hemodialysis is better than peritoneal dialysis). Exchange transfusion is not useful.

- Alternative excretory pathways for ammonia: a loading dose of $250 \mathrm{mg} / \mathrm{kg}$ is followed by $250-400 \mathrm{mg} / \mathrm{kg} /$ day in 4 divided doses. L-Arginine-200 mg/kg/day, L-carnitine (oral/IV)— $200 \mathrm{mg} / \mathrm{kg} /$ day.

Management of suspected organics acidemia:

- Stop feeds and intravenous $10 \%$ glucose (up to $1.5 \times$ maintenance)

- Supportive care: hydration, treatment of sepsis, seizures, ventilation

- Carnitine: $200 \mathrm{mg} / \mathrm{kg} /$ day in 3 divided doses IV or oral

- Treatment of acidosis: sodium bicarbonate $0.35-0.5 \mathrm{mEq} / \mathrm{kg} /$ hour (max 1-2)

- Cofactors: biotin 10 mg/day orally, vitamin B12 1-2 mg/day IM, thiamine $100 \mathrm{mg} /$ day, riboflavin $100 \mathrm{mg} /$ day

- If hyperammonemia present, treat with sodium benzoate

- Metronidazole-to-reduce organic acid by gut flora

\section{References}

1. Ghosh S, Basu A. Acute encephalitis syndrome in India: the changing scenario. Ann Neurosci 2016;23(3):131-133. DOI: 10.1159/00044 9177.

2. John TJ, Verghese VP, Arunkumar G, et al. The syndrome of acute encephalitis in children in India: need for new thinking. Indian J Med Res 2017;146(2):158-161. DOI: 10.4103/ijmr.IJMR_1497_16.

3. Karmarkar SA, Aneja S, Khare S, et al. A study of acute febrile encephalopathy with special reference to viral etiology. Indian J Pediatr 2008;75(8):801-805. DOI: 10.1007/s12098-008-0150-2.

4. Tandel K, Sharma S, Dash PK, et al. Emergence of human West Nile Virus infection among pediatric population in Madhya Pradesh, India. J Med Virol 2019;91(3):493-497. DOI: 10.1002/jmv.25325.

5. Tiwari JK, Malhotra B, Chauhan A, et al. Aetiological study of viruses causing acute encephalitis syndrome in North West India. Indian J Med Microbiol 2017;35(4):529-534. DOI: 10.4103/ijmm.IJMM_17_180.

6. Jain P, Prakash S, Tripathi PK, et al. Emergence of Orientia tsutsugamushi as an important cause of acute encephalitis syndrome in India. PLoS Negl Trop Dis 2018;12(3):e0006346. DOI: 10.1371/journal. pntd.0006346.

7. Kumar R, Kumar P, Singh MK, et al. Epidemiologicalprofile of acute viral encephalitis. Indian J Pediatr 2018;85(5):358-363. DOI: 10.1007/ s12098-017-2481-3.

8. Bloch KC, Glaser CA. Encephalitis surveillance through the emerging infections program, 1997-2010. Emerg Infect Dis 2015;21(9):1562-1567. DOI: 10.3201/eid2109.150295.

9. Simon DW, Da Silva YS, Zuccoli G, et al. Acute encephalitis. Crit Care Clin 2013;29(2):259-277. DOI: 10.1016/j.ccc.2013.01.001.

10. Kneen R, Michael BD, Menson E, et al. Management of suspected viral encephalitis in children - association of British neurologists and British pediatric allergy immunology and infection group national guidelines. J Infect 2012;64(5):449-477. DOI: 10.1016/j.jinf.2011.11.013.

11. Ravi V, Mani R, Govekar S, et al. Aetiology and laboratory diagnosis of acute encephalitis syndrome with special reference to India. J Commun Dis 2014;46(1):12-23.

12. Sharma S, Mishra D, Aneja S, et al. Consensus guidelines on evaluation and management of suspected acute viral encephalitis in children in India. Indian Pediatr 2012;49(11):897-910. DOI: 10.1007/s13312-0120216-0.

13. Marais $\mathrm{S}$, Thwaites $\mathrm{G}$, Schoeman JF, et al. Tuberculous meningitis: a uniform case definition for use in clinical research. Lancet Infect Dis 2010;10(11):803-812. DOI: 10.1016/S1473-3099(10)70138-9.

14. Murthy JM. Tuberculous meningitis: the challenges. Neurol India 2010;58(5):716-722. DOI: 10.4103/0028-3886.72178.

15. Mizuguchi $M$, Abe J, Mikkaichi K, et al. Acute necrotising encephalopathy of childhood: a new syndrome presenting with multifocal, symmetric brain lesions. J Neurol Neurosurg Psychiatry 1995;58(5):555-561. DOI: 10.1136/jnnp.58.5.555.

16. Okumura A, Mizuguchi $M$, Kidokoro $H$, et al. Outcome of acute necrotizing encephalopathy in relation to treatment with corticosteroids and gammaglobulin. Brain Dev 2009;31(3):221-227. DOI: 10.1016/j.braindev.2008.03.005.

17. Wong AM, Simon EM, Zimmerman RA, et al. Acute necrotizing encephalopathy of childhood: correlation of MR findings and clinical outcome. Am J Neuroradiol 2006;27(9):1919-1923.

18. PohID,AlperG, Van Haren K, etal. Acutedisseminatedencephalomyelitis updates on an inflammatory CNS syndrome. Neurology 2016;87(9 Suppl 2):S38-S45. DOI: 10.1212/WNL.0000000000002825.

19. Tenembaum S, Chitnis T, Ness J, et al. Acute disseminated encephalomyelitis. Neurology 2007;68(16 Suppl 2):S23-S36. DOI: 10.1212/01.wnl.0000259404.51352.7f.

20. Graus F, Titulaer MJ, Balu R, et al. A clinical approach to diagnosis of autoimmune encephalitis. Lancet Neurol 2016;15(4):391-404. DOI: 10.1016/S1474-4422(15)00401-9.

21. Dalmau J, Lancaster E, Martinez-Hernandez E, et al. Clinical experience and laboratory investigations in patients with anti-NMDAR encephalitis. Lancet Neurol 2011;10(1):63-74. DOI: 10.1016/S14744422(10)70253-2.

22. Payer J, Petrovic T, Lisy L, et al. Hashimoto encephalopathy: a rare intricate syndrome. Int J Endocrinol Metab 2012;10(2):506-514. DOI: 10.5812/ijem.4174.

23. Odaka M, Yuki N, Yamada M, et al. Bickerstaff's brainstem encephalitis: clinical features of 62 cases and a subgroup associated with GuillainBarré syndrome. Brain 2003;126(Pt 10):2279-2290. DOI: 10.1093/brain/ awg233.

24. Gaspard N, Hirsch LJ, Sculier C, et al. New-onset refractory status epilepticus (NORSE) and febrile infection-related epilepsy syndrome (FIRES): State of the art and perspectives. Epilepsia 2018;59(4):745752. DOI: 10.1111/epi.14022.

25. Serino $D$, Santarone ME, Caputo $D$, et al. Febrile infection-related epilepsy syndrome (FIRES): prevalence, impact and management strategies. Neuropsychiatr Dis Treat 2019;15:1897-1903.DOI: 10.2147/ NDT.S177803.

26. Takanashi J. Two newly proposed infectious encephalitis/ encephalopathy syndromes. Brain Dev 2009;31(7):521-528. DOI: 10.1016/j.braindev.2009.02.012.

27. Fukui KO, Kubota M, Terashima H, et al. Early administration of vitamins B1 and B6 and I-carnitine prevents a second attack of acute encephalopathy with biphasic seizures and late reduced diffusion: a case control study. Brain Dev 2019;41(7):618-624. DOI: 10.1016/j.braindev.2019.02.015.

28. Christopher R, Bindu PS. An insight into the biochemistry of inborn errors of metabolism for a clinical neurologist. Ann Indian Acad Neurol 2008;11(2):68-81. DOI: 10.4103/0972-2327.41873.

29. Bosilkovski M. Brucellosis: Clinical manifestations, diagnosis, treatment and prevention 2019, accessed August 18, 2019, available from https://www.uptodate.com.

30. Rathi N, Kulkarni A, Yewale V, et al. IAP guidelines on rickettsial diseases in children. Indian Pediatrics 2017;54(3):223-229. DOI: 10.1007/s13312-017-1035-0.

31. Centers for Disease Control and Prevention (CDC). Naegleria fowleri-primary amebic meningoencephalitis (PAM). Available at: www.cdc.gov/parasites/naegleria/treament.html, accessed Sept 6, 2019.

32. Centers for Disease Control and Prevention (CDC). Acantamoebagranulomatous amoebic encephalitis (GAE). Available at: www.cdc. 
gov/parasites/acanthamoeba/treatment.html, accessed Sept 6, 2019.

33. Jain S, Patel B, Bhatt GC. Enteroviral encephalitis in children: clinical features, pathophysiology, and treatment advances. Pathog Glob Health 2014;108(5):216-222. DOI: 10.1179/2047773214Y.000000 0145 .
34. Kumar R, Basu A, Sinha S, et al. Role of oral minocycline in acute encephalitis syndrome in India - a randomized controlled trial. BMC Infect Dis 2016;16:67. DOI: 10.1186/s12879-016-1385-6.

35. Revised National Tuberculosis Control Programme and Indian Academy of Pediatrics. RNTCP updated pediatric TB guidelines 2019. Available at https://tbcindia.gov.in, accessed Sept 4, 2019.

\section{Algorithm: Diagnostic Approach to AES}

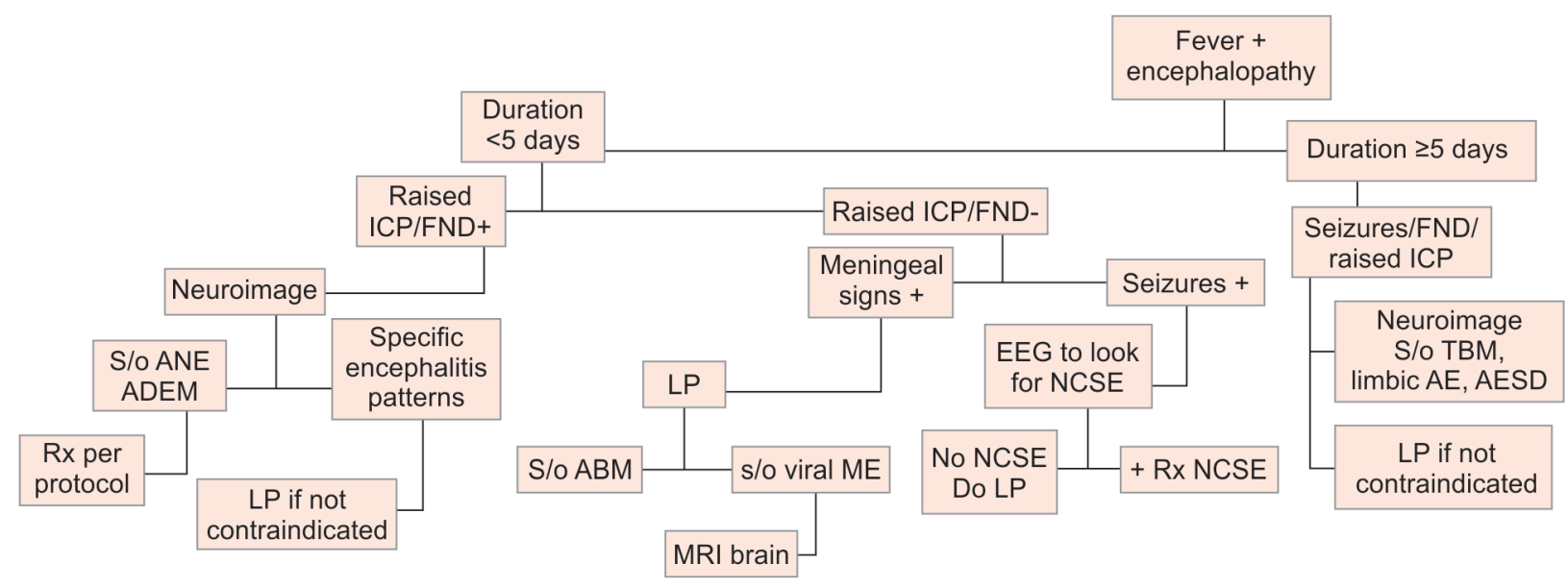

Clues to specific diagnosis in AES

\begin{tabular}{|c|c|c|}
\hline Diagnostic entity & Clues on history/examination & Laboratory investigations \\
\hline \multirow[t]{2}{*}{ Viral meningoencephalitis } & Geographic trends & MRI \\
\hline & Rapid progression often with seizures/raised ICP & CSF-multiplex PCR \\
\hline \multirow{2}{*}{$\begin{array}{l}\text { Complicated pyogenic } \\
\text { meningitis }\end{array}$} & \multirow{2}{*}{$\begin{array}{l}\text { Evolving neurologic signs, toxic look with meningeal } \\
\text { signs }\end{array}$} & CSF \\
\hline & & CT/MRI \\
\hline \multirow[t]{3}{*}{ Rickettsial } & \multirow[t]{3}{*}{ Typical vasculitic rash involving palms/soles, eschar } & PCR on blood samples \\
\hline & & Serology \\
\hline & & Response to doxycycline \\
\hline Cerebral malaria & Endemic area/travel, seizure often status, anemia & Smear for MP \\
\hline Dengue & Seasonal, facial blanching rash, narrow pulse pressure & $\begin{array}{l}\text { NS1 Antigen, antibody after } 5 \text { days, } \\
\text { thrombocytopenia }\end{array}$ \\
\hline Enteric & $\begin{array}{l}\text { Rash, splenomegaly, mild encephalopathy, } \\
>1 \text { week fever }\end{array}$ & Blood culture \\
\hline \multirow[t]{3}{*}{ Tubercular } & \multirow{3}{*}{$\begin{array}{l}\text { Fever }>5 \text { days, meningeal signs, cranial nerve palsies, } \\
\text { h/o contact, evidence of TB elsewhere }\end{array}$} & CSF-NAAT for TB, AFB culture \\
\hline & & $\mathrm{CT} / \mathrm{MRI}$ \\
\hline & & GA for $A F B, C X R$ \\
\hline \multirow[t]{2}{*}{ ADEM } & \multirow{2}{*}{$\begin{array}{l}\text { Encephalopathy with multifocal neurologic deficits of } \\
\text { acute/subacute onset often with recent febrile illness/ } \\
\text { vaccination }\end{array}$} & Neuroimaging \\
\hline & & Biomarkers-MOG, NMO IgG in serum \\
\hline \multirow[t]{2}{*}{ ANE } & \multirow{2}{*}{$\begin{array}{l}\text { Abrupt onset of encephalopathy, raised ICP, tone } \\
\text { abnormalities with/without autonomic dysfunction }\end{array}$} & Typical findings on MRI \\
\hline & & Liver transaminases \\
\hline \multirow[t]{3}{*}{ Autoimmune encephalitis } & \multirow{3}{*}{$\begin{array}{l}\text { Acute/subacute onset neuropsychiatric illness with } \\
\text { movement disorder, sleep disturbance }\end{array}$} & MRI \\
\hline & & CSF-antibodies \\
\hline & & Serum antibodies \\
\hline \multirow[t]{4}{*}{ Neurometabolic disorders (IEM) } & \multirow[t]{4}{*}{ Recurrent encephalopathy/family history } & High anion gap metabolic acidosis, ketosis \\
\hline & & Hyperammonemia, Persistent hypoglycemia \\
\hline & & TMS/GCMS \\
\hline & & Neuroimaging \\
\hline
\end{tabular}

